\title{
Resolution Limit Measured by Fourier Transform: 0.61 Angstrom Information Transfer through HAADF-STEM
}

\author{
Y. Peng, A. Y. Borisevich, A. R. Lupini, and S. J. Pennycook \\ Condensed Matter Sciences Division, Oak Ridge National Laboratory, Oak Ridge, TN 37831-6031
}

Achieving higher resolution has been an enduring pursuit for electron microscopists. An unexpected consequence of improved resolution in scanning transmission electron microscopy (STEM) is the decreased depth of focus, resulting in a changeover from a projection mode of imaging to a depth slicing mode of imaging with increasing illumination angle [1]. Recently with an aberration-corrected VG Microscope's 300 kV HB603U STEM, pairs of Si columns $0.78 \AA$ apart in Si [112] (Fig. 1a) have been directly resolved at ORNL [2]. Fig. 1b shows the modulus of the Fourier transform of the image; there is weak information transfer to the (804) $0.61 \AA$ spacing, which we define as the measured resolution limit.

To justify the use of the Fourier transform to determine the resolution limit, Fig. 1c compares the Fourier transform of a simulated high-angle annular dark field (HAADF) or Z-contrast image to the Fourier transform of the probe used for the Bloch wave simulations. Excellent agreement is seen for a thin crystal where ideal incoherent imaging applies, and the information transfer envelope is determined by the probe. Diffractograms can thus be used to evaluate the resolution limit. Thicker crystals show reduced high frequency transfer, but no spurious sum or difference frequencies occur. The 000 reflection increases with increasing thickness due to increased absorption and background, while the subsidiary peaks generally decrease steadily, except the kinematically forbidden beams such as 222 (Fig. 2a).

Next, frozen phonon simulations using experimentally measured parameters are attempted to match the experimental data. The diffractogram data is extracted from the raw image using a 2-dimensional Gaussian fit, and the 000 reflection is ignored since it is a background related DC offset. The ratio between the two strongest reflections (111 and 220) is connected with the sample thickness, which can thus be determined by matching the ratio. The broadening effect of the source size is taken into account by convolving the simulated image with a model function. As the analysis turns out, the best match (Fig. 2b) occurs at a sample thickness of $100 \AA$ and a source size modeled by a Gaussian of $0.31 \AA$ FWHM. Dynamical scattering cannot fully account for the observation of two strong 222 and 402 reflections which would have been kinematically forbidden by lattice symmetry, and the significant discrepancy can be attributed to surface corrugation and possible surface reconstruction [3]. Fig. 3a shows the simulated image with random noise included for a more realistic description, and the profile compares well with the experimental data (Fig. 3b). The experimental probe has an expected size in the range of $0.6 \AA$, but noise and other equipment related instabilities form the limit to achieving higher resolution. Further simulations demonstrate that, at a $6 \%$ ratio of Poisson noise mean to maximum signal, the 804 reflection which marks the lower limit of detectable information transfer would sink to the noise level.

\section{References:}

[1] Y. Peng, P. D. Nellist, and S. J. Pennycook, J. Electron Microsc. 53 (2004) 257.

[2] P. D. Nellist et al., Science 305 (2004) 1741.

[3] K. N. Tu and A. Howie, Phil. Mag. B 37 (1978) 73; Y. Tanishiro et al., Surf. Sci. 10 (1982) 135.

[4] This work was supported by the USDOE under contract DE-AC05-00OR22725 managed by UTBattelle, LLC. 

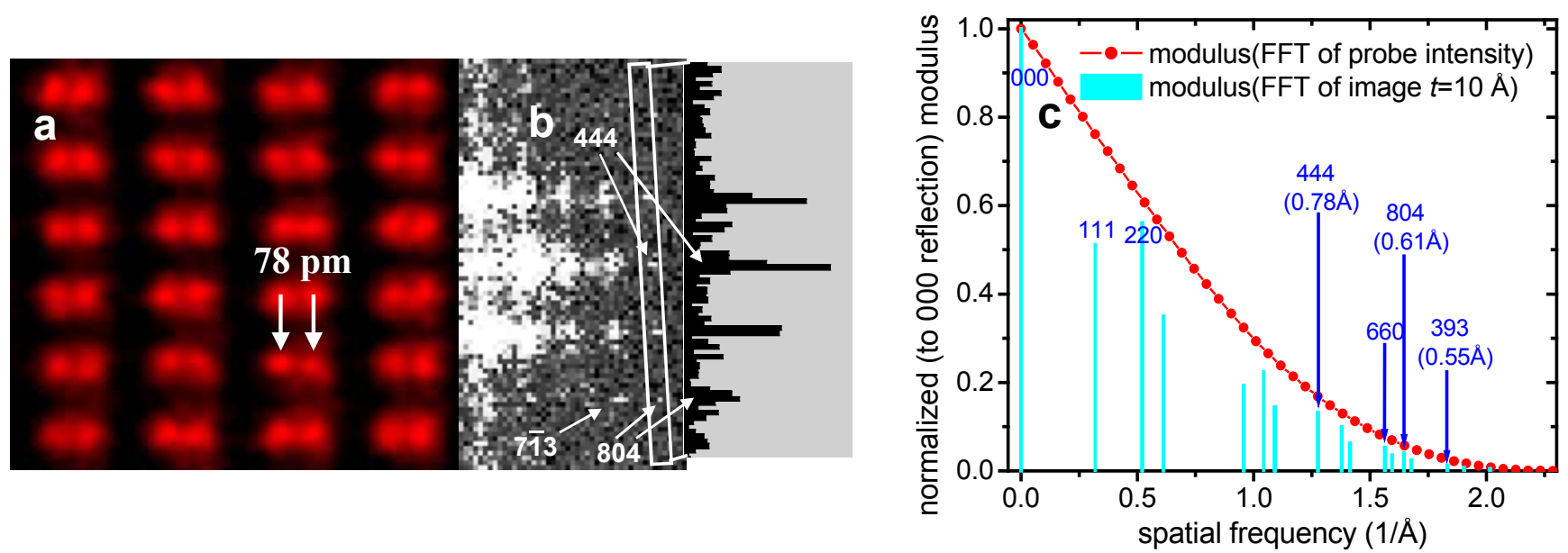

FIG. 1: (a) Z-contrast image (has been low-pass filtered to reduce noise and unwarped to remove image drift) of Si [112] resolving the $0.78 \AA$ dumbbell, with (b) Fourier transform indicating information transfer to $0.61 \AA$ (804). (c) Fourier transforms of a simulated image and the probe used for the simulation.
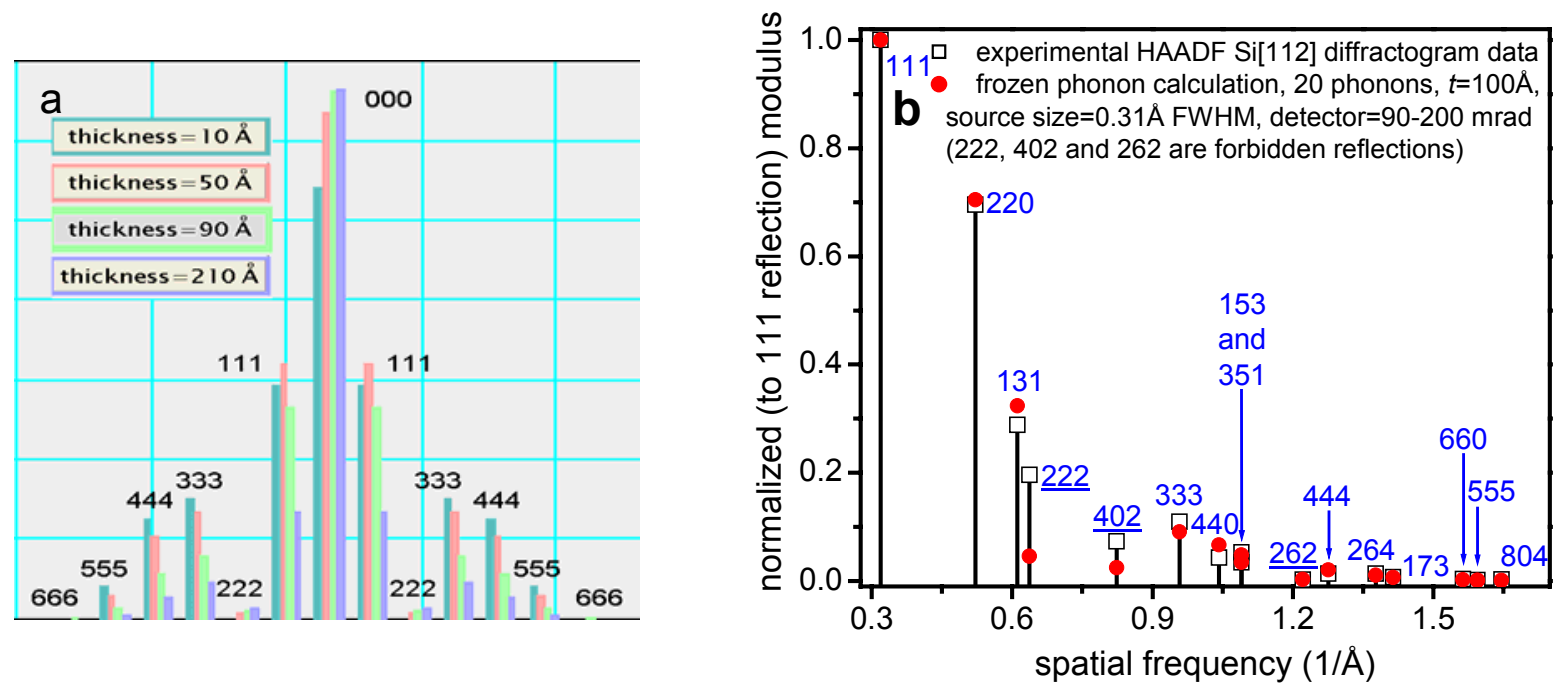

FIG. 2: (a) linescan of simulated diffractograms for Si [112] of varied thickness. (b) Frozen phonon calculation compared to experimental diffractogram. Simulation parameters: accelerating voltage $=300 \mathrm{kV}$, probe semi-angle $=22 \mathrm{mrad}, \mathrm{Cs}=-0.037 \mathrm{~mm}, \mathrm{C} 5=100 \mathrm{~mm}$, detector $=90-200 \mathrm{mrad}, \mathrm{Cc}=1.6 \mathrm{~mm}, \mathrm{FWHM}$ energy spread $=0.3 \mathrm{eV}$, central defocus $=20 \AA$.
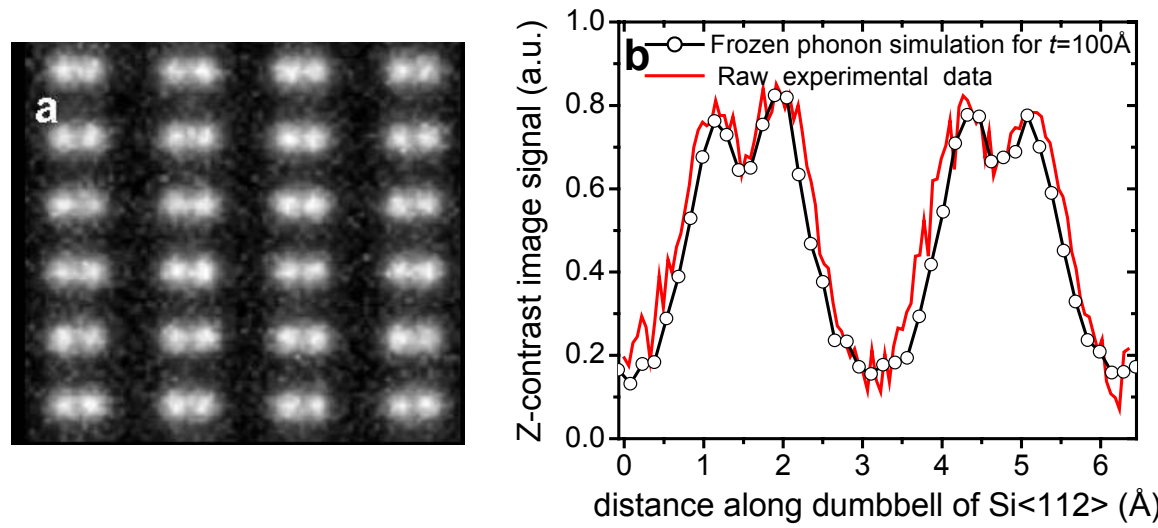

FIG. 3: (a) Simulated Z-contrast image containing random noise modeled by Poisson distribution with a mean equal to $4 \%$ of the maximum image intensity. (b) An intensity profile through two column pairs in (a). An experimental profile is shown for comparison. 